\title{
Hydrodynamic Simulation of an Orbital Shaking Test for the Degradation Assessment of Blood-Contact Biomedical Coatings
}

Zuo-Syuan Dong ${ }^{1, a}$, Chau-Chang Chou ${ }^{1,2, b^{*}}$, Chi-Hsio Yeh ${ }^{3,4, c}$, Yu-Heng Pan ${ }^{1, d}$

${ }^{1}$ Department of Mechanical and Mechatronic Engineering, National Taiwan Ocean University

${ }^{2}$ Center for Marine Mechatronic Systems (CMMS), National Taiwan Ocean University

${ }^{3}$ College of Medicine, Chang Gung University

${ }^{4}$ Division of Thoracic and Cardiovascular Surgery, Chang Gung Memorial Hospital

ag00172032@gmail.com, bcchou@ntou.edu.tw, cyehccl@cgmh.org.tw, ${ }^{\text {d }}$ seohyun81714@amail.com

Keywords: Mechanical Heart Valve, Computational Fluid Dynamics, Degrar on, Biom ical Coatings

Abstract. Biomedical coatings are used to promote the wear resistance an hecom at foility of a mechanical heart valve (MHV). An orbital shaking test was propos a to ass the dr ability of the coatings by the amount of eroded material due to the surrour a fluid. Ho er, the shaker's rotating conditions and the corresponding physiological cond on th till lack of understanding. This study implemented numerical simulations by establis ing fluid dy i model to evaluate the intensity of the shear stress under various rotating speed and diameter of the shaker. The results are valuable to conduct in vitro tests for estimating the pe prmance of piomedical coatings under real hemodynamic conditions and can be applied to other flu contact in plants.

\section{Introduction}

Mechanical heart valve (MHV) is widely us in the replacement of human heart valve and ventricular assist devices. In orde to luce th failure after valve transplantation, there is a biocompatible coating to modify pros Leses' su ace [1]. During the serving time of an MHV in vivo, its coating is constantly s cess caused by the blood flow. In general, when the valves are moving from op ing to clo the average flow rate is greater than $0.8 \mathrm{~m} / \mathrm{s}$ due to the venturi effect $[2,3,4]$. $\mathrm{Tb}$ bi mpatible atings may lose their function and even be removed from the surface, of which ne ntensh the shear stress and the duration are two major factors that cause the long-term degradation of the co ngs.

To examine adhe on strength between biocompatible coatings and MHV, the samples with collagen-heparin oosite ating were immersed in containing tubes filled with physiological saline, in ed at ${ }^{\circ} \mathrm{C}$ and then shaken on an orbital shaker for different durations [5]. The remain a ma ial on substrate was measured and compared. It is a simple and easy way to evalua he performance of any biomedical coating, organic or inorganic, on the blood contact de However, the shaker's rotating conditions, including the rotating speed and diameter, and the corre. Ading physiological condition was still lack of understanding.

In this work, an orbital shaker is chosen for simulating the fluid condition in heart and blood vessels, the remained amount of coated heparin after the experiment is regarded as indicators of the binding at different rotating speeds and diameters. A preliminary analysis of the biocompatible coatings degradation in dynamics condition is presented, and a previous experimental work was also investigated [6].

\section{Materials and Method}

Problem Description. Fig. 1(a) shows a commercially available orbital shaker. The container on the orbital shaker being translated along the path is illustrated in Fig. 1(b), where (x, y), ( $\left.x^{\prime}, y^{\prime}\right), R$, and $\omega$ 
represent the fixed global coordinate, the orbitally-moving coordinate on the container, the radius of rotating path, and the rotating speed. As the sample and the fluid inside the container were both moving with the path in the same rotating speed, a simulation model was established by exploiting moving boundary technique of the computational domain.

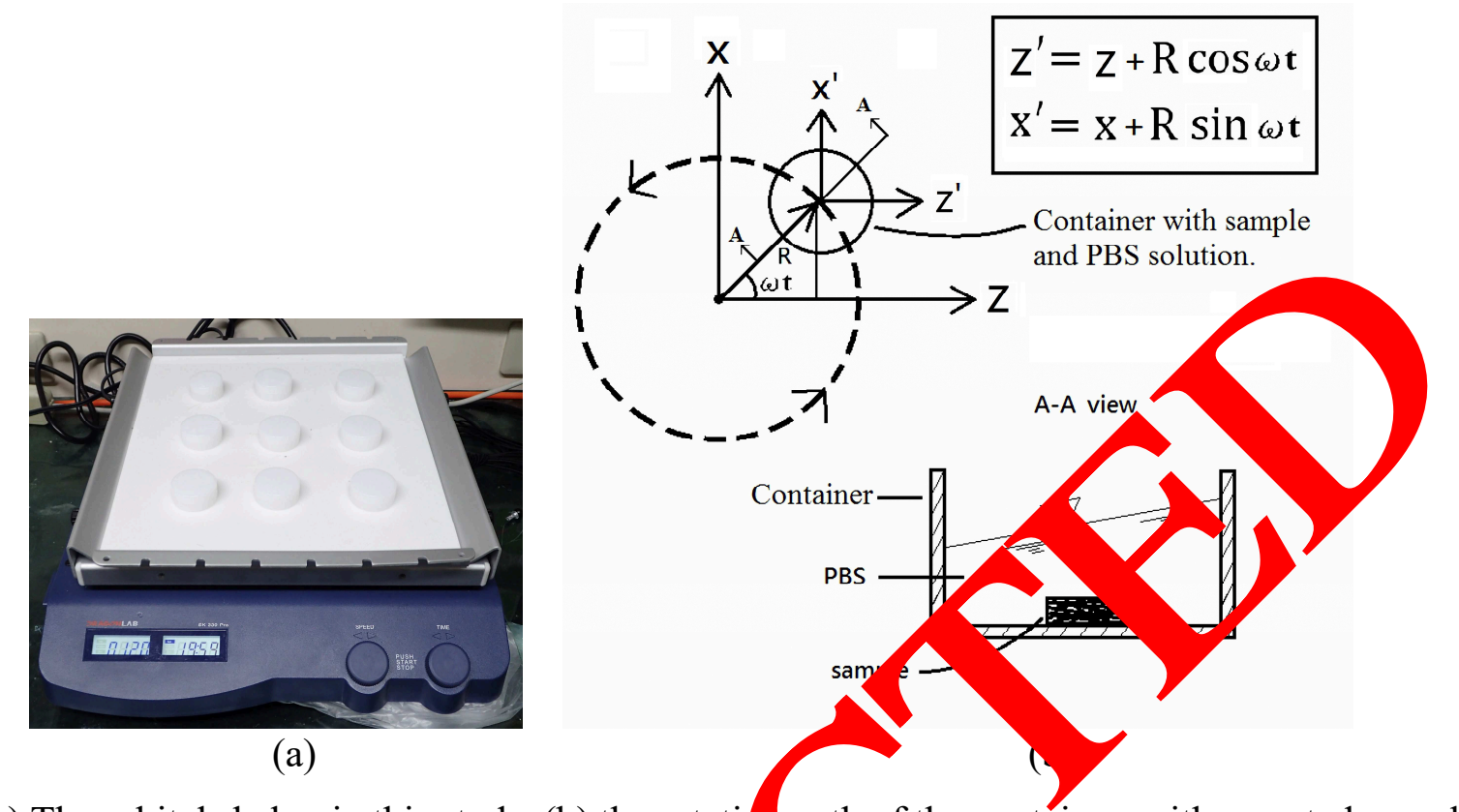

Fig. 1 (a) The orbital shaker in this study, (b) the rotati path of the sontainer, with a coated sample and PBS solution, and the relation equation of the red globa coordinate $(x, y, z)$ and the orbitally-moving coordinate ( $\left.x^{\prime}, y, z^{\prime}\right) . \omega$ is the rotating $s_{\mathrm{A}}$. path in $\mathrm{mm}$, and $\mathrm{t}$ is the time in second. The A- $A$ is the cross section of the container to illustrate the detail status of the fluid.

Simulation setup. In this model, 110 mputat nal area was composed by two domains, the surrounding air domain and the tain domain the dimensions and material properties of the domains are summarized in $\mathrm{T}$

The simulation was carr f out with commercial computational fluid dynamic (CFD) software. The fluid had a multipha e p erty and a epsilon turbulence model was applied. The solution was modelled as a mixtum wato nd air, which was incompressible, viscous, and isothermal. By means of this mod, the shear flo as accessed in the flow.

Rotating spe and d yeter of the shaker were two independent variables in the simulation. The rotating speed or shaker as varied from $60 \mathrm{rpm}$ to $300 \mathrm{rpm}$ and the rotating diameter of the shaker wa m, 3 a nd 5 cm respectively.

Table 1 Properties of the surrounding air domain.

\begin{tabular}{ll} 
Diameter $[\mathrm{mm}]$ & 100 \\
Height $[\mathrm{mm}]$ & 20 \\
Density $\left[\mathrm{kg} / \mathrm{m}^{3}\right]$ & 1.225 \\
Viscosity $[\mathrm{kg} /(\mathrm{s} \cdot \mathrm{m})]$ & 0.00001789 \\
\hline
\end{tabular}

Table 2 Properties of the container domain.

\begin{tabular}{ll}
\hline Diameter $[\mathrm{mm}]$ & 30 \\
Height $[\mathrm{mm}]$ & 20 \\
Liquid level $[\mathrm{mm}]$ & 10 \\
Air Density $\left[\mathrm{kg} / \mathrm{m}^{3}\right]$ & 1.225 \\
Solution Density $\left[\mathrm{kg} / \mathrm{m}^{3}\right]$ & 998.2 \\
Solution Viscosity $[\mathrm{kg} /(\mathrm{s} \cdot \mathrm{m})]$ & 0.001003 \\
\hline
\end{tabular}




\section{Result and Experimental application}

In this section we present the results of our numerical simulations for five rotating speeds and three rotating diameters of the shaker. Fig. 2 demonstrates a typical trajectory and liquid distribution at four positions where the rotating speed and diameter are $120 \mathrm{rpm}$ and $1 \mathrm{~cm}$.

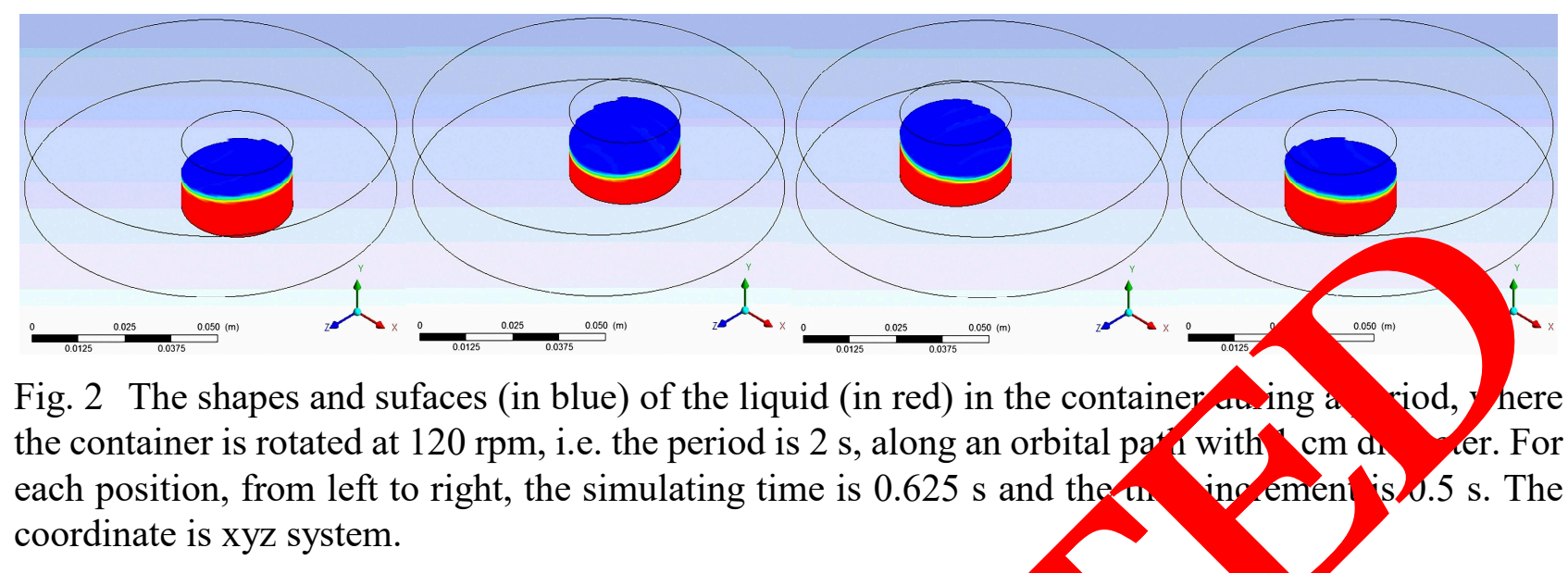

Dynamic performance in the rotational speed and diameter va tion. To o cuate the variable hydrodynamic condition in terms of the erosion from the flo a ting he surface of an MHV, the instantaneous viscous shear stresses can be obtained by th CFD model es Mshed in this work.

After a very short transient procedure, the flow insi the container was proven, from period to period, to be steady and well-posed at any specific pos of the or ital path as indicated in Fig. 2. The distributions of the shear stress vector for three rot diame ers on the bottom surface at the simulating time of $2.5 \mathrm{~s}$ are visualized in Fig. 3 isometrio and top view. The rotating speed of these cases were kept at $120 \mathrm{rpm}$. The avera an aximum shear stresses along an arbitrary diameter on the bottom surface of the container enow in Fig. 4. The distributions of the shear stress are similar, which suggests that otating iameter has little effect on the shear stress of the bottom surface.

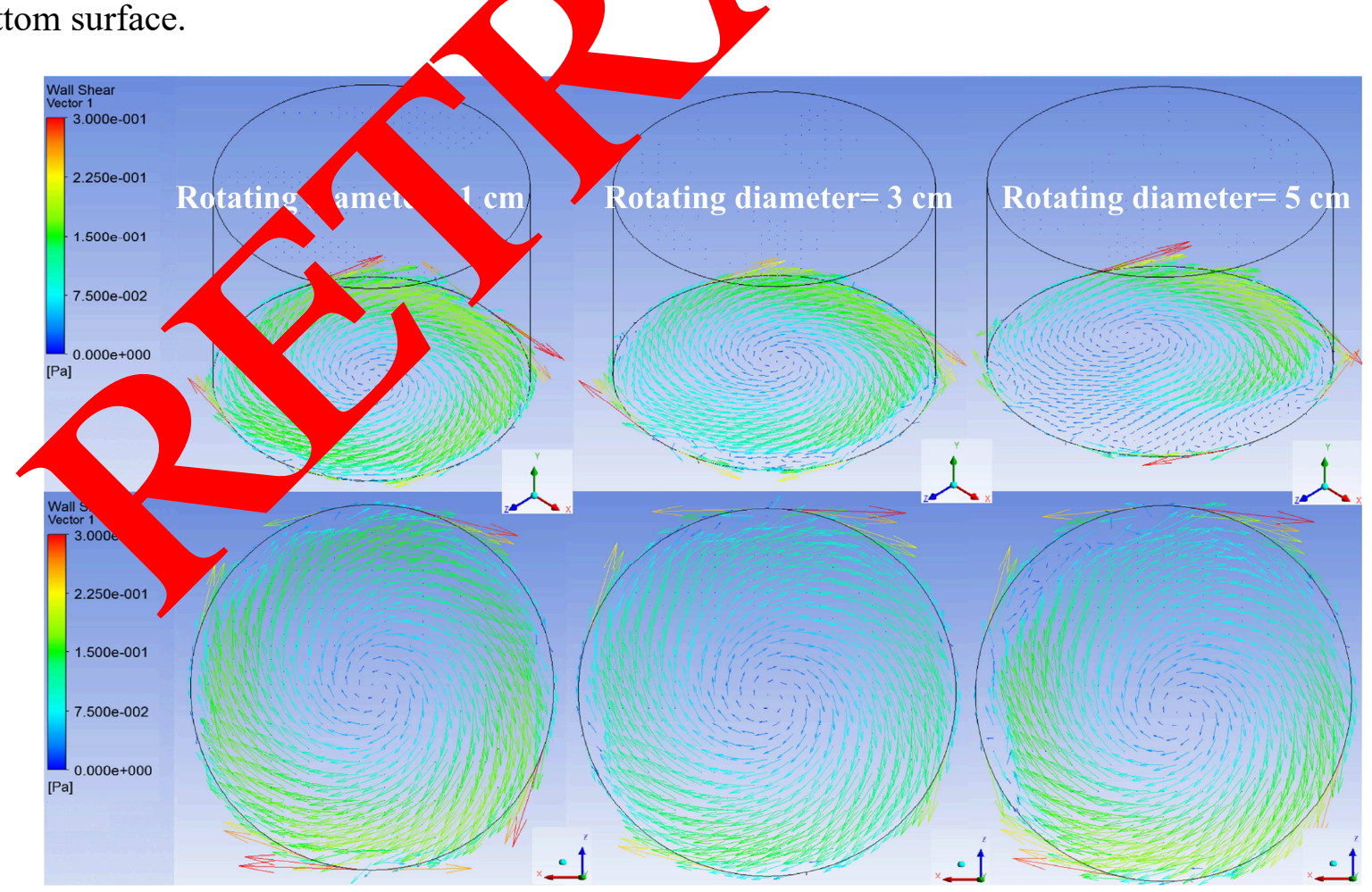

Fig. 3 The isometric view (upper) and top view (lower) of the shear stress distributed on the bottom surface of the container. The shaker's rotational speed is $120 \mathrm{rpm}$ and the rotational diameter is $1 \mathrm{~cm}$ (left), $3 \mathrm{~cm}$ (middle) and $5 \mathrm{~cm}$ (right), respectively. The coordinate is x'yz' system. 

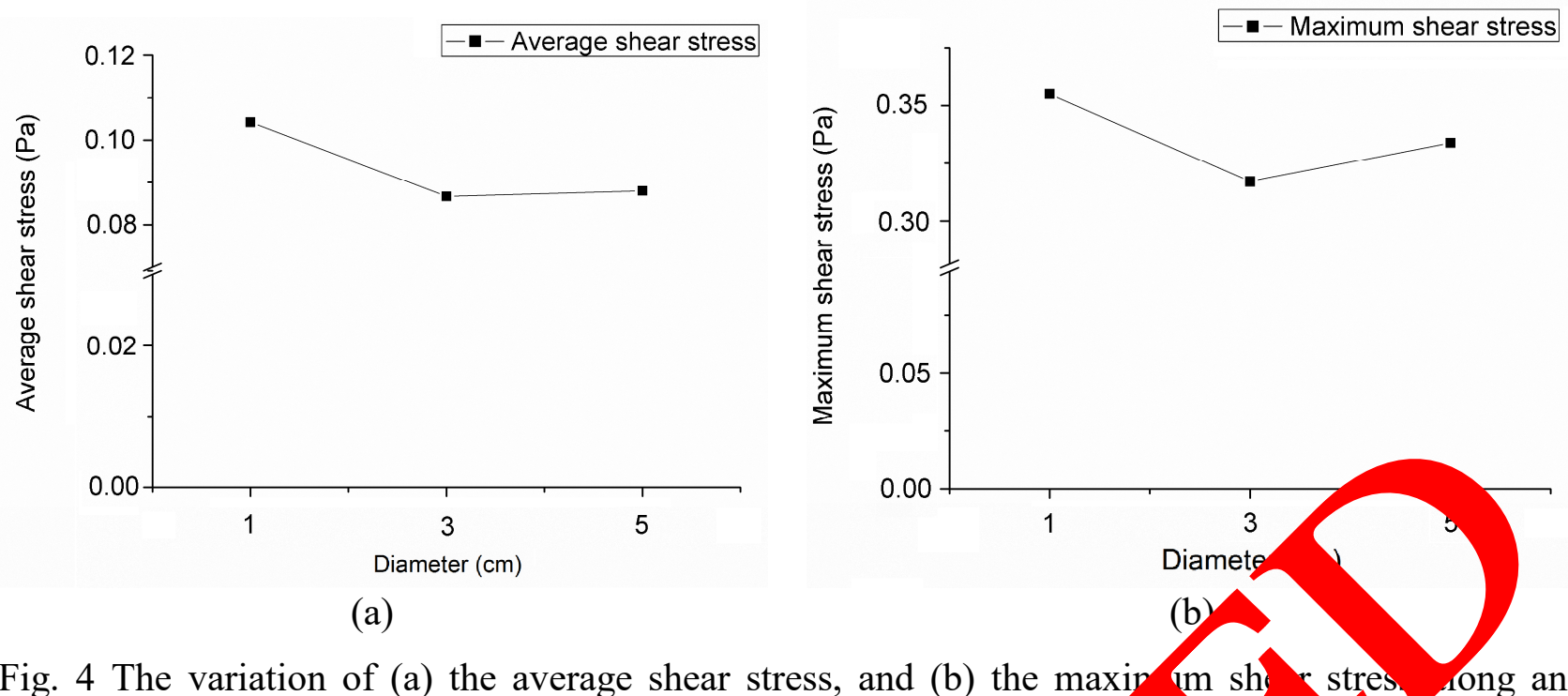

(a)

Fig. 4 The variation of (a) the average shear stress, and (b) the maxip $m$ sh stres $r$ ong an arbitrary diameter on the bottom surface of the container with three oto o cramet s? (Rotating speed $=120 \mathrm{rpm})$.

In Fig. 5, the distributions of the shear stress vector for three tatin. at the simulating time of $2.5 \mathrm{~s}$ are also illustrated by is view diameter of these cases were kept at $1 \mathrm{~cm}$. The average st ess and maximum stress are shown in Fig. 6. It is worthy of notification that the trend of the stresses i nore signifig ant than the those in Fig. 4 with the rotating diameters, especially during the rotating sp betweer 180 to $240 \mathrm{rpm}$. Therefore, the rotating speed of orbital shaker is suggested to k the major level of the long-term erosion test in this study.

Experimental application. In the experimental si dy, we present the degradation assessment of two coatings by the application of orbi $a \mathrm{Sh}_{\mathrm{H}}$. Thes samples were both grade 2 titanium substrates treated with dopamine, poly-L, of then eoated with heparin/collagen multilayers and a heparin outmost layer. Electr on tic r-oy-rager technique was conducted to build these coatings and the amount of hepari vere meas by toluidine blue $O$ test [6]. The samples with 4 and

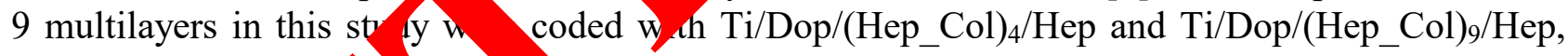
respectively.

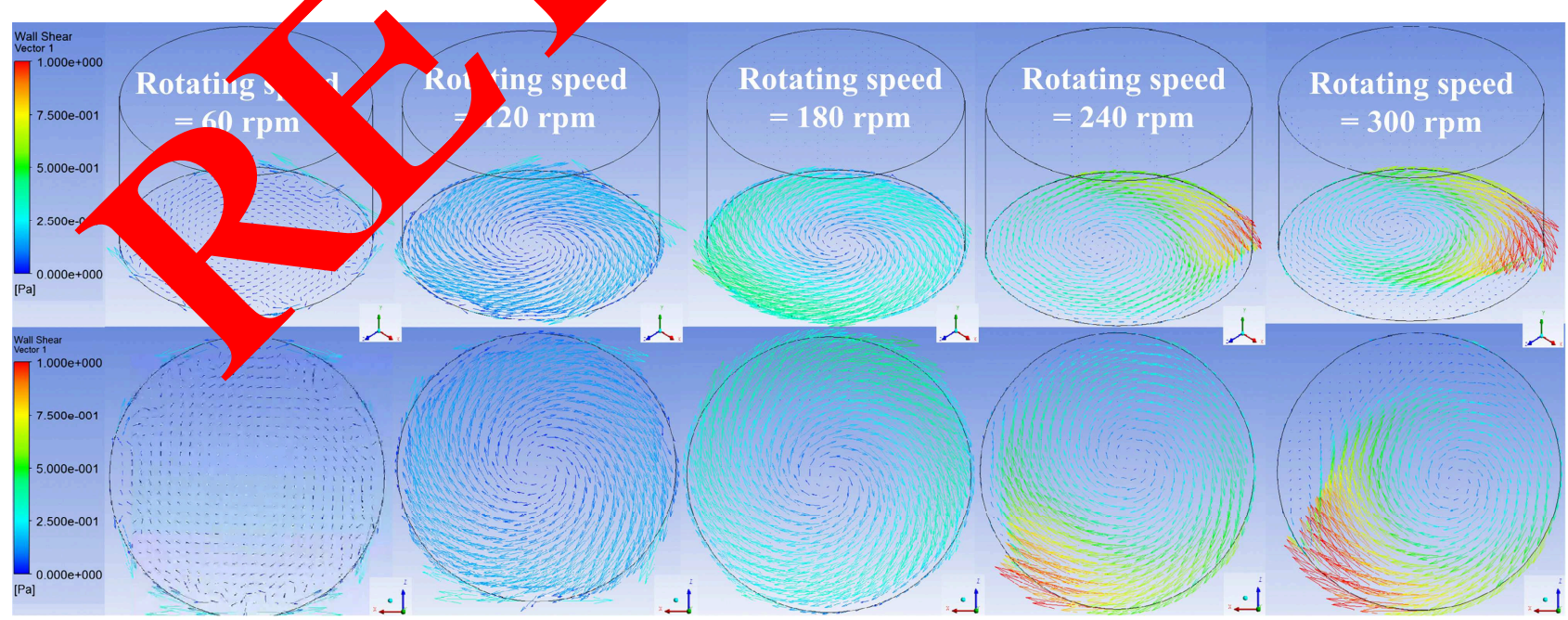

Fig. 5 The isometric view and top view of the shear stress distribution on the bottom surface of the container. The shaker's rotational diameter is fixed at $1 \mathrm{~cm}$ and the rotational speed is adjusted to $60 \mathrm{rpm}, 120 \mathrm{rpm}, 180 \mathrm{rpm}, 240 \mathrm{rpm}$ and $300 \mathrm{rpm}$ respectively from left to right. The coordinate is x'yz' system. 


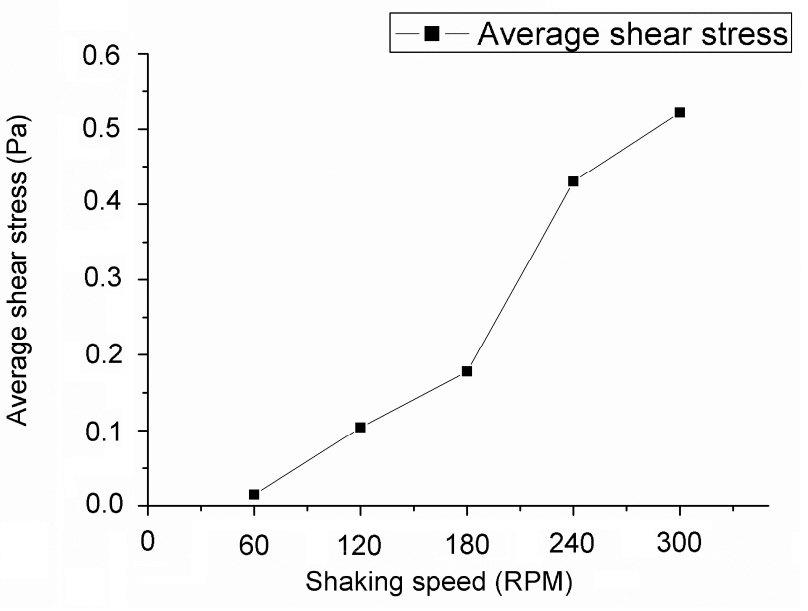

(a)

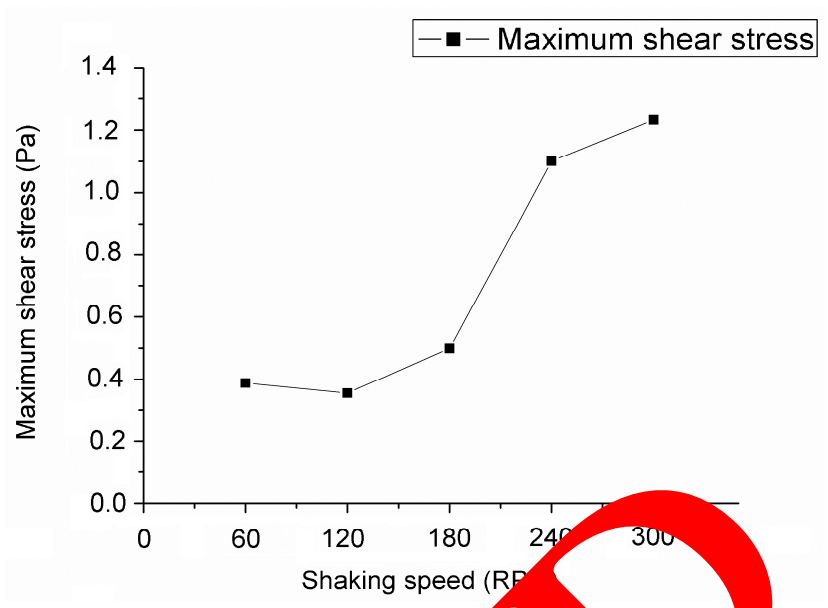

(b)

Fig. 6 The variation of (a) the average shear stress, and (b) the maxim a shar str an an arbitrary diameter on the bottom surface of the container with five rotatin eeds cot 2 tin diameter $=1 \mathrm{~cm})$.

Fig. 7 shows the quantitative analysis of the coatings by the man heparin hrom the beginning to the fifteen days. The rotating speed of the orbital shal as kept $20 \mathrm{rpm}$ and the rotating diameter, in $1 \mathrm{~cm}$. It can be observed that the heparin/col/gen coatings wer significantly removed by the fluid for the first two days. The samples with 9 multi yers, even hoving nearly the same heparin at the beginning, could maintain higher heparin content. $\mathrm{t}$ vever, wh $\mathrm{n}$ the tests exceeded four days, the degradation of both coatings reached a low and steady according to the numerical results in this study, the averas stress and maximum shear stress actuated on the coatings were 0.1 and $0.4 \mathrm{~Pa}$, which can be sse san compared with the real physiological conditions.

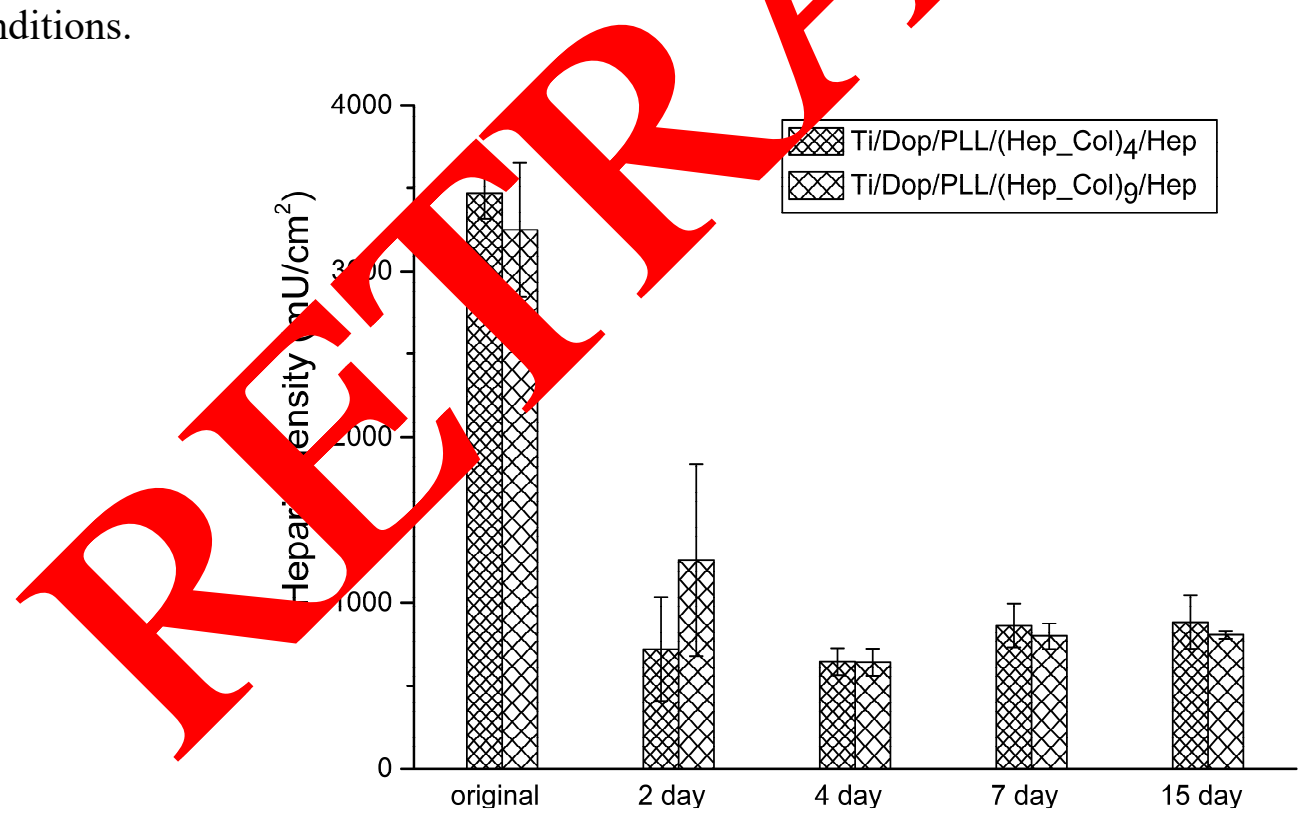

Fig. 7 Heparin density of two samples, Ti/Dop/(Hep_Col $)_{4} /$ Hep and Ti/Dop/(Hep_Col) $)_{9} / \mathrm{Hep}$, from the beginning to the 15 th days of the tests conducted by the orbital shaker $(\mathrm{N}=3)$.

\section{Summary}

As the degradation of the biomedical coatings are caused by the shear stress and the actuating duration, this work provides a numerical evaluation of the shear stress in the container of an orbital shaker to investigate the mechanism of the degradation. The rotational speed of the shaker is verified 
to be the major operating parameter that can change the shear stress of the flow in the container. By applying the results to the experimental investigation, the correlation of biomedical coating's degradation in the fluid-contact conditions and its shear stress levels was demonstrated.

\section{References}

[1] J. Chen, Q. Li, J. Chen, C. Chen and N. Huang: Appl. Surf. Sci., Vol.255 (2009), pp 6894-6900

[2] C.-K. Huang and J.-H. Chen: J. Taiwan Soc. Nav. Archi. Mar. Eng., Vol.30 (2011), No.3, pp $171-180$

[3] I. Borazjani and F. Sotiropoulos: J. Biomech. Eng. Trans. ASME, Vol.132 (2010), pp $111005-111012$

[4] S. A. Jones: Ann. Biomed. Eng., Vol.23 (1995), pp 21-28

[5] J. Chen, N. Huang, Q. Li, Ch. H. Chu, J. Li and M. F. Maitz: Appl. Surf. sci., Vol.3 201,, pp. 281-289

[6] C.-C. Chou, S.-W. Hsin, H.-C. Lin, C.-H. Yeh, R. Wu and W.- Tern Surf. C at. Technol., Vol. 303 (2016), Part A, pp.277-282

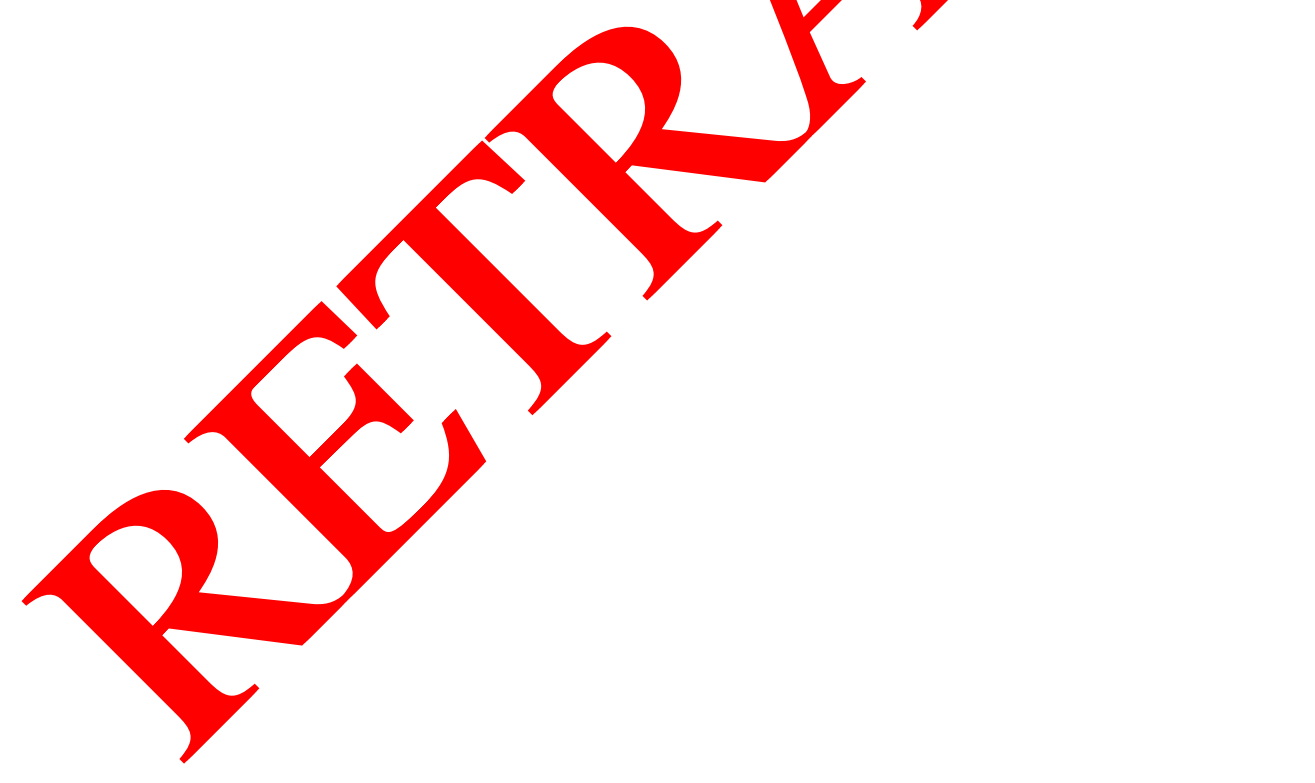

